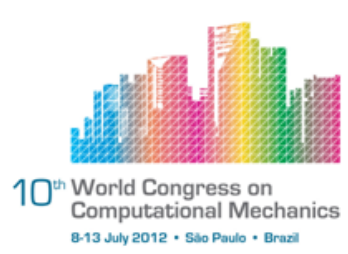

\title{
TESTING OF HIGHER ORDER FINITE ELEMENTS BASED ON LAGRANGE POLYNOMIALS IN DEPENDENCE OF THE UNDERLYING NODAL GRID
}

\author{
D. Schmicker ${ }^{1}$, J.M. Vivar-Perez ${ }^{2}$, U. Gabbert ${ }^{3}$, \\ ${ }^{1}$ Faculty of Mechanical Engineering, Otto-von-Guericke University Magdeburg \\ (david.schmicker@ovgu.de) \\ ${ }^{2}$ Faculty of Mechanical Engineering, Otto-von-Guericke University Magdeburg \\ (juan.vivar@gmail.com) \\ ${ }^{3}$ Faculty of Mechanical Engineering, Otto-von-Guericke University Magdeburg \\ (ulrich.gabbert@ovgu.de)
}

\begin{abstract}
The paper deals with the investigation of a higher order finite element method utilizing Lagrangian interpolation polynomials as shape functions, also often referred to as spectral element method (SEM). The main concern is to analyze the influence of the underlying nodal distribution on the convergence, performance and stability properties of the solution. To this end four different nodal configurations are investigated, namely the equispaced grid (EQ), the Gauss point grid (GP), the Gauss-Lobatto-Legendre grid (GLL) and the Chebyshev-Gauss-Legendre (CGL) grid. It is concluded that the nodal distribution does not alter the convergence behavior directly. Differences are only observed in cases, where, e.g., the approximation of the geometry of distorted elements or the implementation of Dirichlet boundary conditions takes place. It is figured out that the EQ-grid cannot be recommended because of the occurrence of Runge's oscillations and very high condition numbers, while the other three nodal configurations show an excellent solution quality and behave almost equal. Further tests have revealed that the solution quality of the GLL-element is not significantly affected by the well known mass lumping technique even if the element has a strongly distorted geometry. This makes the GLL-grid favourable in dynamic analyses.
\end{abstract}

Keywords: Higher order finite element method, Spectral element method, Convergence studies

\section{INTRODUCTION}

The finite element mesh as well as the applied shape functions decisively affect the quality of the numerical solution of a given field problem. In general, there are two basic options in achieving a well approximated solution of the field equations. At first, the geometry is subdivided into very small finite elements of low order shape functions, which results in a 
huge amount of finite elements (h-refinement). At second, a comparatively coarser mesh of finite elements with higher order shape functions is used ( $p$-refinement). The letter $h$ represents the elements size and $p$ constitutes the polynomial degree of the shape functions. Whereas in the early beginning of applying finite elements the $h$-method was used almost exclusively, in the 1980s p-methods were investigated and applied increasingly - a development which is closely related to the works of Babuška, Szabó (see [1] to [4]). The construction of finite elements with higher order polynomials is by no means unique as there are various suggestions in doing this. Well known approaches are, e.g., the application of Legendre-polynomials resulting in the hierarchical p-FEM $[1,7,8]$, the utilization of non-uniform rational B-splines (NURBS) corresponding to the N-FEM $[9,10]$ and the application of Lagrange-polynomials often referred to as Spectral Element Method (SEM) [11, 12, 13]. The latter term should not be mixed up with the likewise named analytical or semianalytical approaches in [5] and [6], for instance. Hence, for the sake of clarity in the following the term SEM is not used anymore. Instead of that the higher-order finite element approaches with Lagrangian polynomials are referred to as HOLP.

To create Lagrangian polynomials a certain grid of nodes in the domain of definition is required. Besides the trivial choice of an equispaced distribution of nodes, various other suggestions of nodal placements are proposed in the literature, such as the Gauss-LobattoLegendre points [12, 14], the Gauss points [13] or the Chebyshev-Gauss-Lobatto points [11, 15]. The aim of this paper is to figure out how the solution of a problem is altered by the underlying nodal distribution of the Lagrangian polynomials. The following study is performed at rather simple linear-elastomechanic problems. Therein the focus is on the convergence rate, the stability and the performance aspects of the respective approaches. In addition, the isoparametric approximation of the element geometry involving the same Lagrangian polynomials as for the shape functions is analyzed and discussed, giving evidence of the benefits and drawbacks of each of the applied nodal distributions.

The paper's outline is as follows. In Section 2 the analyzed variational problem is described. The applied shape functions are given in Section 3. In Section 4 several illustrative model problems are introduced, which are solved and analyzed to answer the above introduced questions. Finally, in Section 6 the results are summarized and recommendations regarding the application of higher order finite elements are given.

\section{THE LINEAR ELASTOMECHANICAL FIELD-EQUATION}

The analyzed partial-differential equation is the Navier-Cauchy equation

$$
(\lambda+\mu) \boldsymbol{\nabla}(\boldsymbol{\nabla} \cdot \boldsymbol{u})-\mu \boldsymbol{\nabla} \otimes(\boldsymbol{\nabla} \otimes \boldsymbol{u})+\rho \boldsymbol{b}=\rho \ddot{\boldsymbol{u}}
$$

with the displacement field $\boldsymbol{u}$, the two Lamé constants $\mu$ and $\lambda$, the mass density $\rho$ and the body forces $\boldsymbol{b}$. The corresponding variational formulation of (1) is the principle of virtual work, stated as

$$
0=-\int_{\mathcal{B}} \delta \boldsymbol{u}^{T} \ddot{\boldsymbol{u}} \rho d V-\int_{\mathcal{B}} \delta \varepsilon^{T} \boldsymbol{C} \boldsymbol{\varepsilon} d V+\int_{\partial \mathcal{B}} \delta \boldsymbol{u}^{T} \boldsymbol{t} d A,
$$

where $\delta \boldsymbol{u}$ is a virtual displacement field fulfilling the Dirichlet boundary conditions, $\boldsymbol{C}$ is the linear stiffness tensor in Voigt's notation, $\delta \varepsilon$ is the virtual strain, and $\varepsilon$ is the strain due to the 
displacements $\boldsymbol{u}$. The latter two relations are given by

$$
\varepsilon=\boldsymbol{D} \boldsymbol{u} \quad \text { and } \quad \delta \varepsilon=\boldsymbol{D} \delta \boldsymbol{u},
$$

with $\boldsymbol{D}$ being a linear operator mapping the displacement field to the strain field. In order to achieve the spatially discretized form of (1) the actual and virtual displacement field $\boldsymbol{u}$ is approximated by

$$
\boldsymbol{u}(\boldsymbol{x}, t)=\boldsymbol{N}(\boldsymbol{x}) \boldsymbol{v}(t) \quad \text { and } \quad \delta \boldsymbol{u}(\boldsymbol{x}, t)=\boldsymbol{N}(\boldsymbol{x}) \delta \boldsymbol{v}(t)
$$

with $\boldsymbol{N}$ containing the space-dependent shape functions, and $\boldsymbol{v}$ is the vector of the timedependent nodal displacements. Arguing that every component of $\delta \boldsymbol{v}$ can be chosen arbitrarily, the spatially discretized form of (1) is derived as

$$
M \ddot{v}+K \boldsymbol{v}=f .
$$

Here, $M$ denotes the mass matrix, $\boldsymbol{K}$ the stiffness matrix, and $\boldsymbol{f}$ the force vector, respectively, as

$$
\boldsymbol{M}=\int_{\mathcal{B}} \rho \boldsymbol{N}^{T} \boldsymbol{N} d V, \boldsymbol{K}=\int_{\mathcal{B}} \boldsymbol{B}^{T} \boldsymbol{C} \boldsymbol{B} d V \text { and } \boldsymbol{f}=\int_{\mathcal{B}} \boldsymbol{t} d A
$$

where $\boldsymbol{B}$ stands for the abbreviation $\boldsymbol{D}(\boldsymbol{N})$. Typically, the shape function matrix $\boldsymbol{N}$ is not defined on the spatial domain, but on a dimensionless reference frame utilizing the isoparametric mapping of the element geometry as

$$
\boldsymbol{x}=\boldsymbol{N}(\boldsymbol{\xi}) \boldsymbol{X},
$$

where the parameters defining the geometry are included in $\boldsymbol{X}$. Moreover, the solution domain is partitioned into small sub-domains, the so called finite elements, resulting in element matrices $\boldsymbol{K}^{(e)}$ and $\boldsymbol{M}^{(e)}$, which have to be assembled to a global system of equations. For more elaborate derivations the reader is referred to standard encyclopedias such as [16] or [18].

\section{LAGRANGE SHAPE FUNCTIONS}

In general, the applied finite element shape functions can be divided into two subgroups. The first group includes unknown parameters $\boldsymbol{v}$, which exhibit a physical interpretation like nodal displacements or angles. The second group of unknowns does not have any physical relevance. An usual approach concerning the non-physical parameters, for instance, are $p$-elements using standardized Legrende polynomials, see [3, 7] and [8], and NURBS, being exploited in [10]. However, the application of Lagrange polynomials belongs to the latter group, since all parameters in $\boldsymbol{v}$ exhibit the interpretation of nodal displacements deriving from the interpolation property of these polynomials. Regarding a polynomial degree $p$, the set of $p+1$ basis shape functions are formally defined as

$$
N_{n}(\xi)=\prod_{j=1, j \neq n}^{p+1} \frac{\xi-\xi_{j}}{\xi_{n}-\xi_{j}}, \quad n=1,2, \ldots,(p+1),
$$

where it is easily comprehended that the shape function values are

$$
N_{j}\left(\xi_{i}\right)= \begin{cases}1 & \text { if } i=j \\ 0 & \text { if } i \neq j\end{cases}
$$


remarking their ability to interpolate a given set of points. To achieve the $C_{0}$-compatibility between the elements the nodal distribution necessarily needs to comprise the boundary locations -1 and +1 . Thus, the utilized grid of the HOLP is defined by the location of the inner nodes of the set $\xi_{n}$. Subsequently, four of such nodal distributions are analyzed, namely:

- Equispaced distribution (EQ):

$$
\xi_{i}^{E Q}=\frac{2(i-1)-p}{p}
$$

- Gauss node distribution (GP):

$$
\xi_{i}^{G P}=\left\{-1, \xi_{0}^{L_{p-1}},+1\right\}
$$

- Gauss-Lobatto-Legendre distribution (GLL):

$$
\xi_{i}^{G L L}=\left\{-1, \xi_{0}^{L o_{p-1}},+1\right\}
$$

- Chebyshev-Gauss-Lobatto distribution (CGL):

$$
\xi_{i}^{C G L}=-\cos \left(\frac{(i-1) \pi}{p}\right) .
$$

Therein $\xi_{0}^{L_{p-1}}$ and $\xi_{0}^{L o_{p-1}}$ are the zeros of the Legendre polynomials

$$
L_{p-1}(\xi)=\frac{1}{2^{p-1}(p-1) !} \frac{d^{p-1}}{d \xi^{p-1}}\left[\left(\xi^{2}-1\right)^{p-1}\right]
$$

or the Lobatto polynomials

$$
L o_{p-1}(\xi)=\frac{1}{2^{p} p !} \frac{d^{p+1}}{d \xi^{p+1}}\left[\left(\xi^{2}-1\right)^{p}\right],
$$

respectively. The EQ-grid as basis of Lagrangian interpolation can not be recommended, because it results in heavy oscillations near the boundaries of the domain. This effect is known as Runge's phenomenon, making the EQ-grid not a suited choice for interpolation tasks. Lagrangian polynomials based on the GP-, GLL- and CGL-grid do not show such a behavior. The GLL-grid is quite popular in applications (see [12, 13, 14] and many more), since in conjunction with the GLL-quadrature rule it enables the possibility to diagonalize the mass matrix through a slight under-integration [13]. Moreover, one can easily see that at the GLL-distribution the shape function values are not only equal to one at their corresponding nodes, but also reach their maxima at these points. Since it can be shown that the CGL-grid ensures minimal errors in the interpolation of smooth functions [17], some authors favor the CGL-based HOLP, as in [11] and [15], for instance. In Fig. 1 the proposed nodal distributions for $p=4$ are displayed for comparison. 


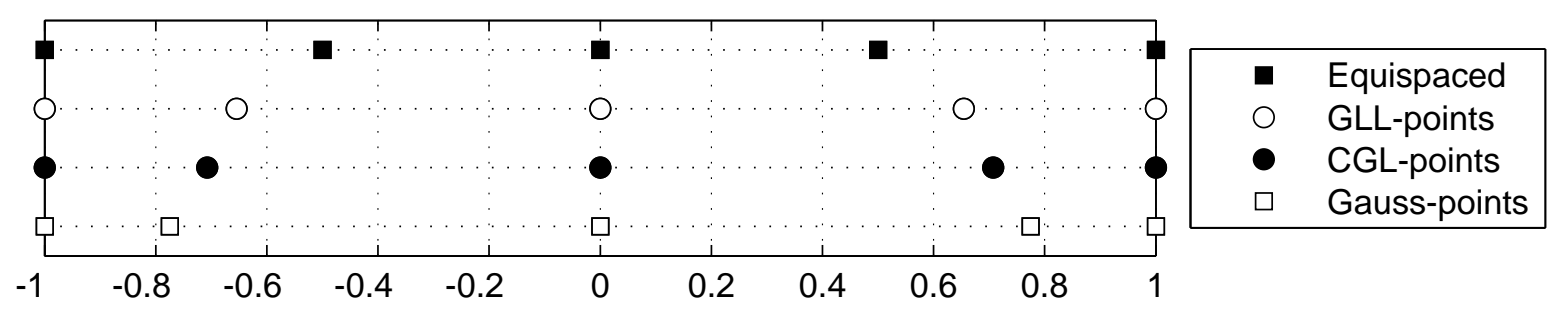

Figure 1. Nodal distribution comparison of degree $p=4$.

\section{ANALYSIS OF THE HIGHER ORDER METHODS}

\subsection{Single undistorted element: The point loaded half-space}

The first problem involving a single undistorted element consists of a point load acting on the center of an elastic half-space, as Figure 2 illustrates. In order to restrict the computational effort, the problem is simplified to a plain stress problem. The thickness is denoted by $t$. Since a point load induces a singularity in the model, which is corrupting the convergence behavior, the point-load is replaced by a static equivalent distributed line load as

$$
q(\xi)=\frac{q_{0}}{1+(b \xi)^{2}}
$$

where $b$ determines the width of the bell shaped curve, and $q_{0}$ is its magnitude. This load has to be distributed to the element nodes by applying eq. (6). ${ }^{1}$ The loading parameters are selected as $b=10$ and $q_{0}=4 \cdot 10^{10}$.

In the following the convergence behaviour is analyzed and presented as a function of the polynomial degree of the shape functions. Since the element has equal dimensions in the $\xi$-direction as well as in the $\eta$-direction, the $p$-refinement will be performed isotropically, meaning that $p_{\xi}=p_{\eta}$. In Figure 3 the von-Mises stresses are displayed for the polynomial degrees $p=2,3,4,5,8$ and 16 of the GLL-distributed Lagrange $p$-element. As anticipated, the maximum equivalent stresses occur in the center of the square near the surface where the line load acts on the body.

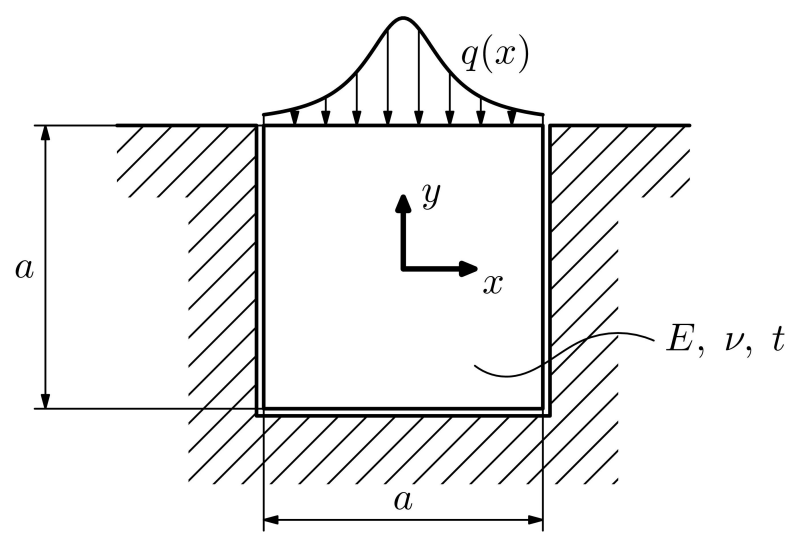

Figure 2. The point loaded half-space $\left(E=70 \cdot 10^{9} ; \nu=0.33 ; a=0.5 ; t=1\right)$.

\footnotetext{
${ }^{1}$ The integral therein will be performed by applying a simple trapezoid rule, since the Gaussian quadrature converges very badly for this specific function.
} 

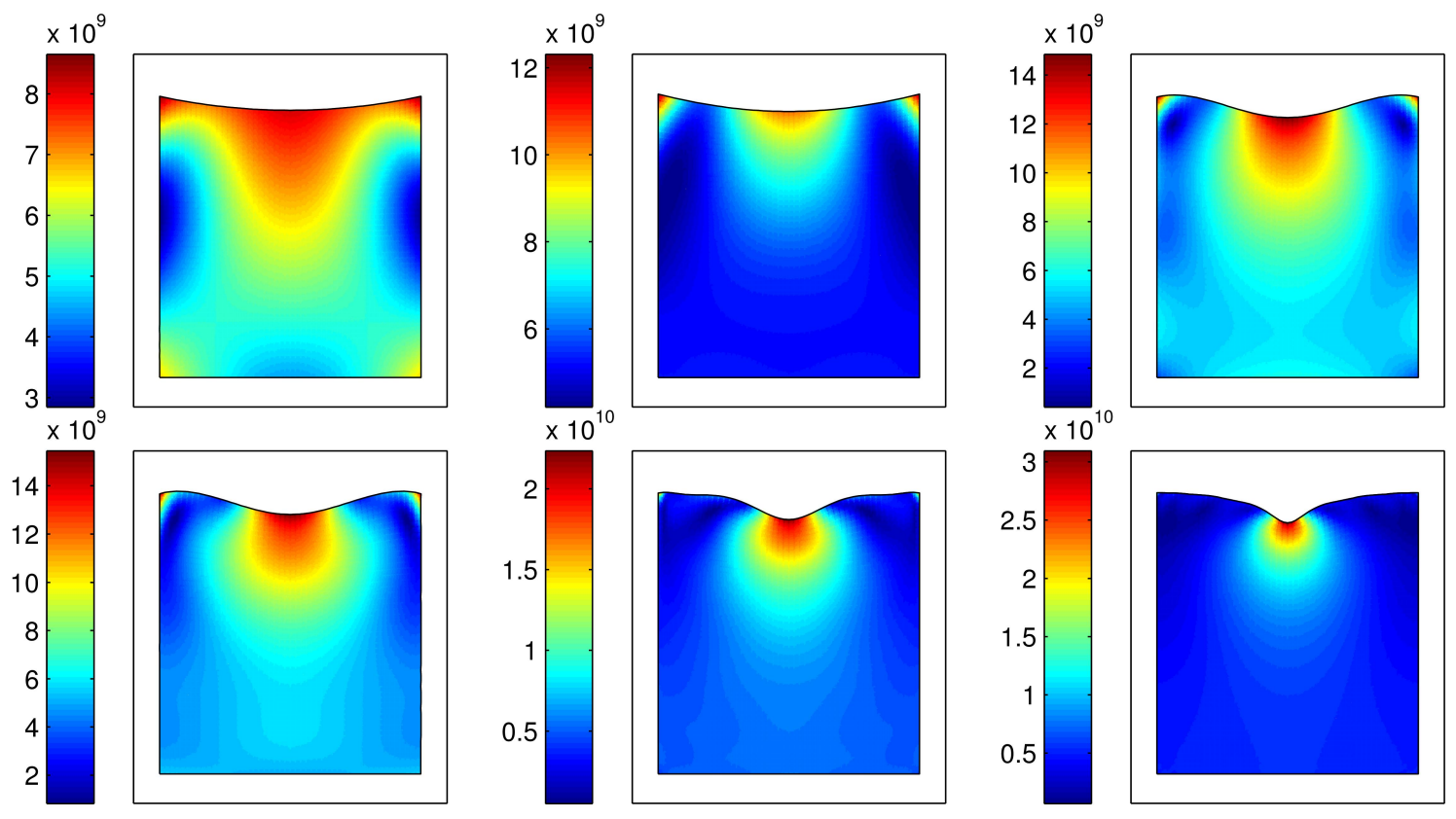

Figure 3. Von-Mises stresses of the point loaded half-space model for the polynomial degrees $p_{\xi}=p_{\eta}=p=2,3,4,5,8$ and 16 utilizing the GLL nodal distribution.

Further, it is analyzed how the nodal distribution influences the solution as well as their convergence behavior. In Figure 4 the displacements of the polynomial shape function degrees $p_{\xi}=p_{\eta}=5$ for all four introduced distributions are displayed. Apparently, the nodal placement has hardly any impact on the solutions, the displacement field approximations nearly coincide ${ }^{2}$. In order to quantify the convergence behavior concerning $p$-refinement the vertical displacement of the point with the coordinates $x=0$ and $y=a / 2$ is observed. For

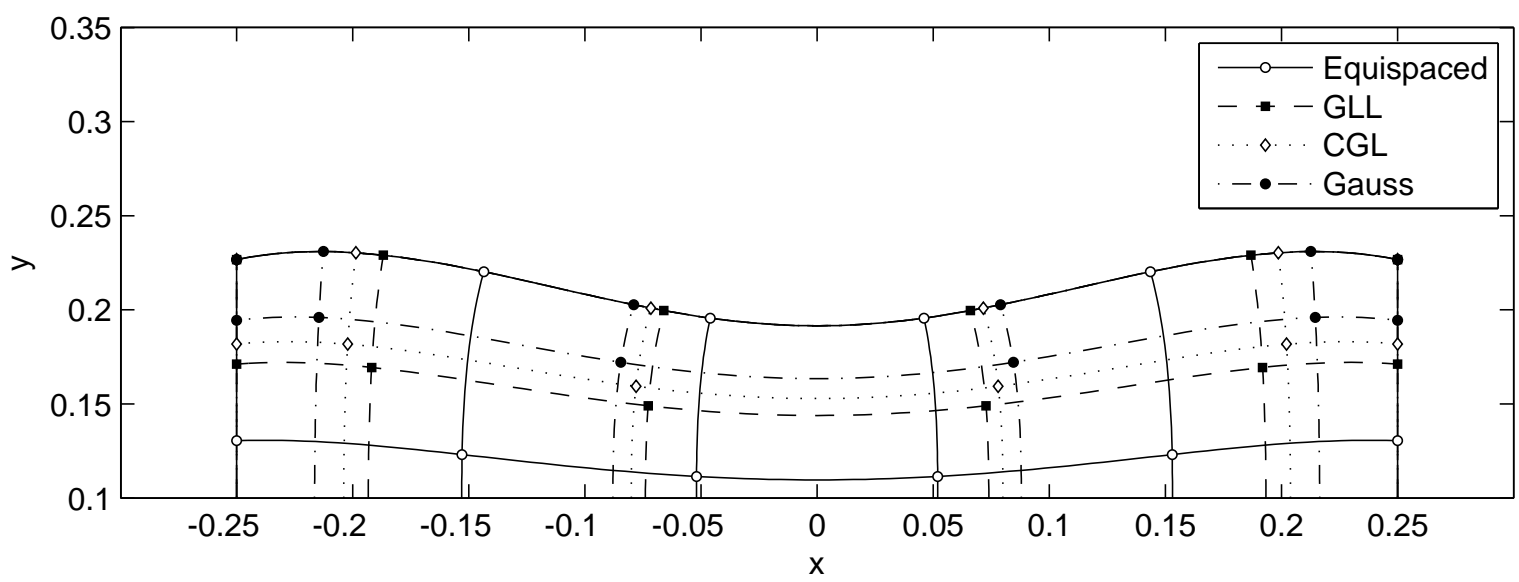

Figure 4. Overlay of the solutions of the point loaded half-space for the polynomial degree $p_{\xi}=p_{\eta}=5$ concerning the four observed nodal distributions.

\footnotetext{
${ }^{2}$ The maximum difference between the displacement field approximations is not greater than $1,05 \cdot 10^{-15}$, which is about $1,8 \cdot 10^{-12} \%$ of the order of magnitude of the displacements.
} 


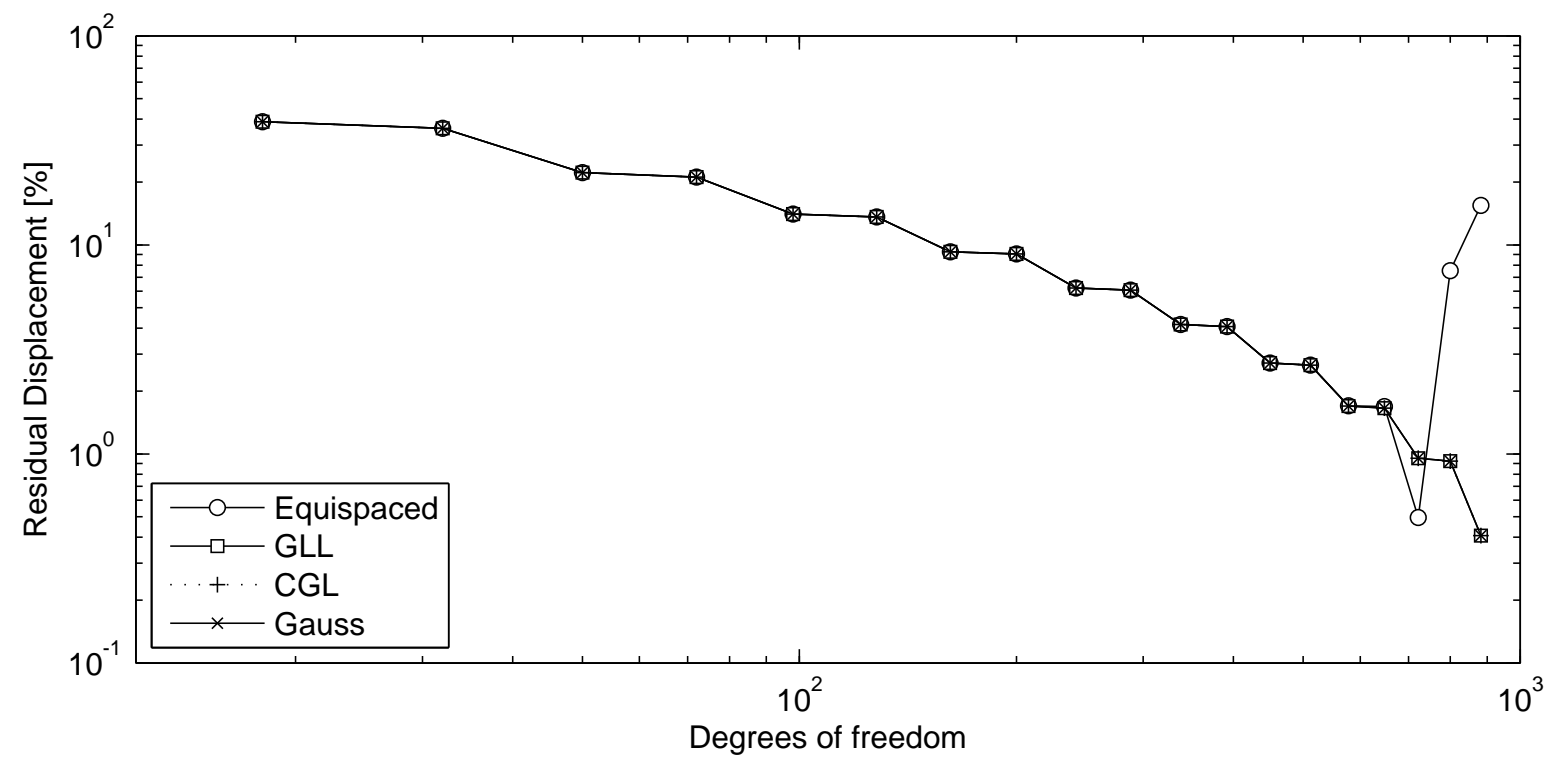

Figure 5. Convergence of the residual vertical displacement of the point with the coordinates $x=0$ and $y=a / 2$ of the point loaded half-space model for the polynomial degrees $p_{\xi}=$ $p_{\eta}=p=2,3,4, \ldots, 20$ concerning the four observed nodal distributions.

the sake being able to plot this value on a logarithmic scale, a pseudo exact ${ }^{3}$ solution gained by the software package Abaqus is used. Therewith a residual value is computed by

$$
\Delta u_{y}=\frac{\left\|u_{y}^{(p)}-u_{y}^{(h)}\right\|}{\left\|u_{y}^{(h)}\right\|}
$$

where $u_{y}^{(p)}$ is the solution of the Lagrange p-element and $u_{y}^{(h)}$ is the Abaqus reference solution.

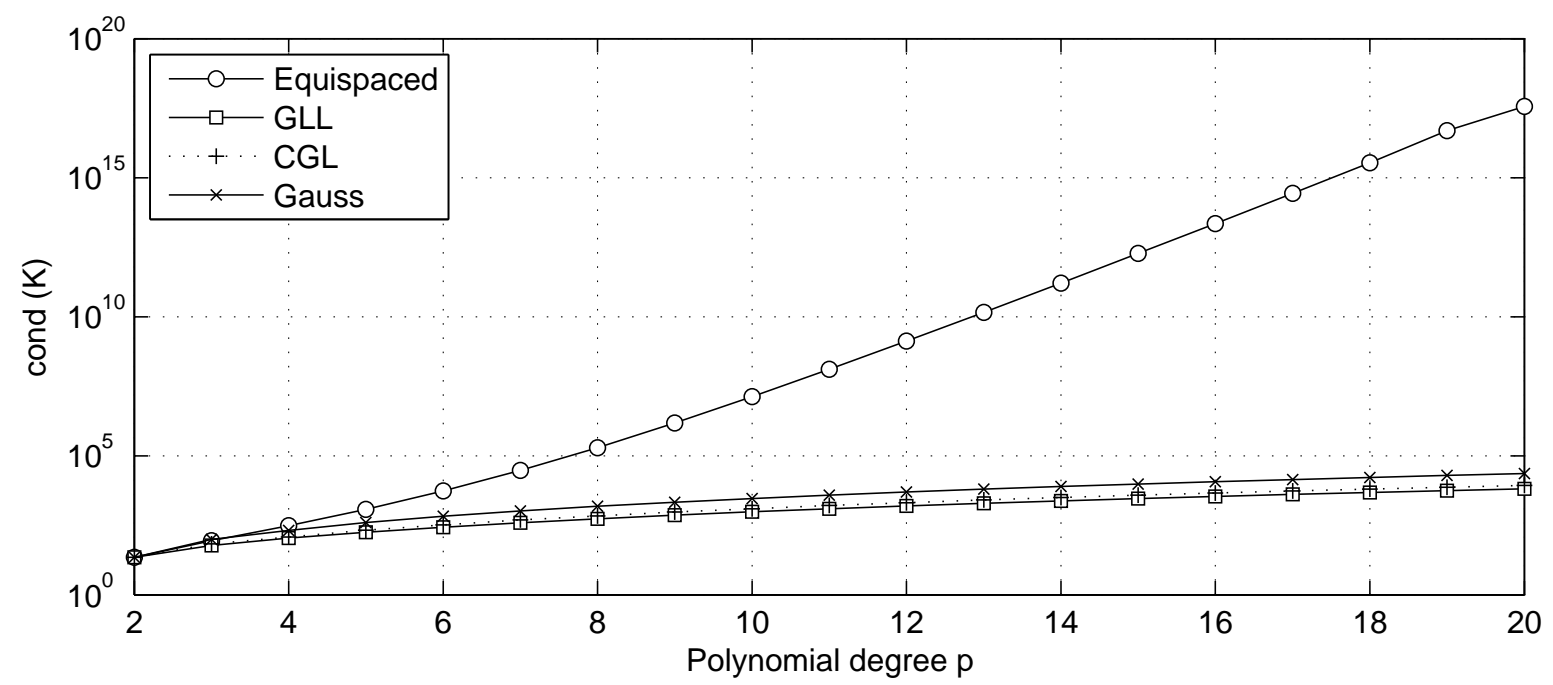

Figure 6. Condition number evolution of the stiffness matrix of the undistorted element based upon the model of the point loaded half-space. The polynomial degree of the shape functions is raised isotropically from $p=p_{\xi}=p_{\eta}=2$ to 20 .

\footnotetext{
${ }^{3}$ Pseudo exact means, that this value is computed with a high number of finite elements ( $h$-refinement). Its solution is calculated with 15625 elements with quadratic shape functions, resulting in a total of 94752 degrees of freedom.
} 
In Figure 5 the amount of the residual displacements is plotted against the resulting degrees of freedom of the model. It is apparent that also at other shape function orders the solution is hardly influenced by the nodal distribution. Hence, the convergence rate does not depend on the nodal basis, but on the chosen order of the Lagrange interpolation polynomials only. Another observed phenomenon is, that for polynomial degrees greater than $p=16$ the equispaced solution drifts off. This is due to the bad condition of the stiffness matrix, which in case of equispaced nodes is orders of magnitudes higher than for the other three analyzed grids, being emphasized in Figure 6.
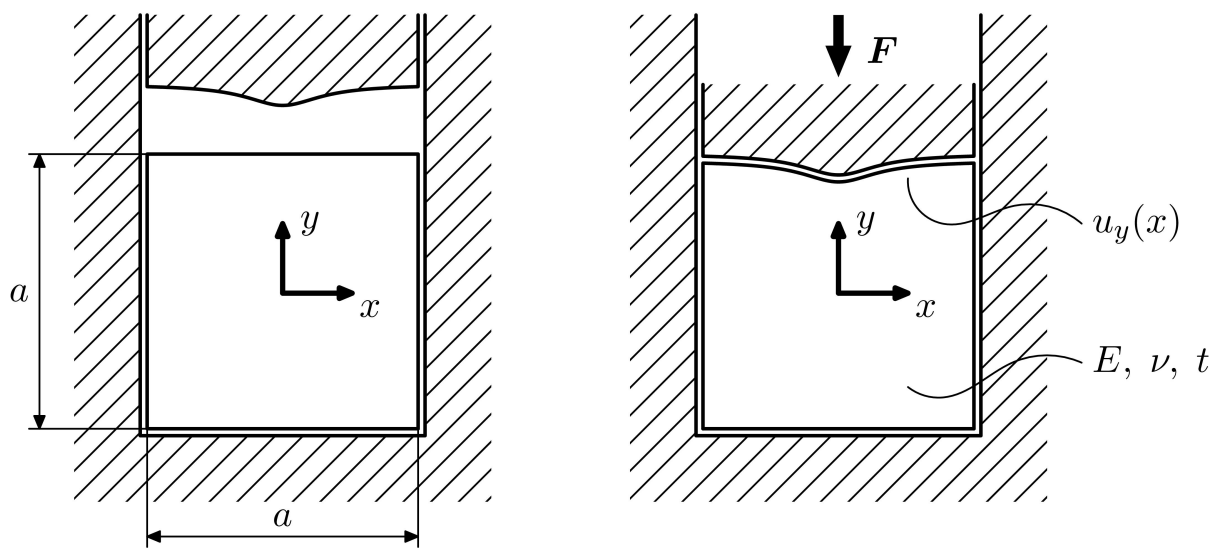

Figure 7. The point loaded half-space with Dirichlet boundary conditions $\left(E=70 \cdot 10^{9}\right.$; $\nu=0.33 ; a=0,5 ; t=1)$.

Subsequently, the problem will be posed in a slight different way. Instead of a Neumann boundary condition a similar Dirichlet boundary condition is prescribed at the top boundary of the square, forcing the displacements as displayed in Figure 7. The prescribed displacements regarding the $y$-direction are assumed as

$$
u_{y}(\xi)=\frac{-u_{0}}{1+(b \xi)^{2}} .
$$

The boundary condition parameters of the following examples are $u_{0}=0.005$ and $b=3$. Figure 8 shows the top boundary of the square including the applied displacements

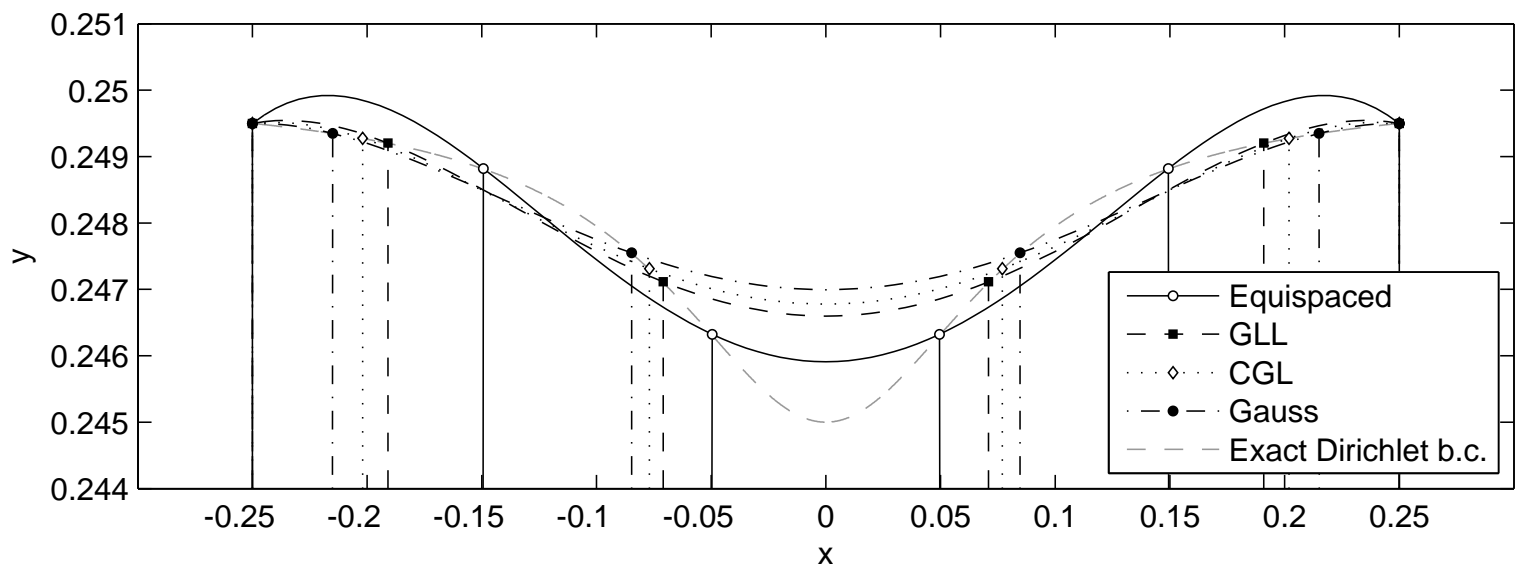

Figure 8. Overlay of the solutions of the point loaded half-space with Dirichlet boundary conditions for the polynomial degree $p_{\xi}=5$. The gray line represents the prescribed Dirichlet displacement. 
of the different types of Lagrangian interpolation polynomials of degree $p=5$. Obviously, the solution is only as good as the shape functions are able to map $u_{y}(\xi)$ correctly. Thus, for this problem the interpolation ability of the ansatz polynomials has a significant impact on the numerical solutions.

\subsection{Single distorted element: The C-Cantilever}

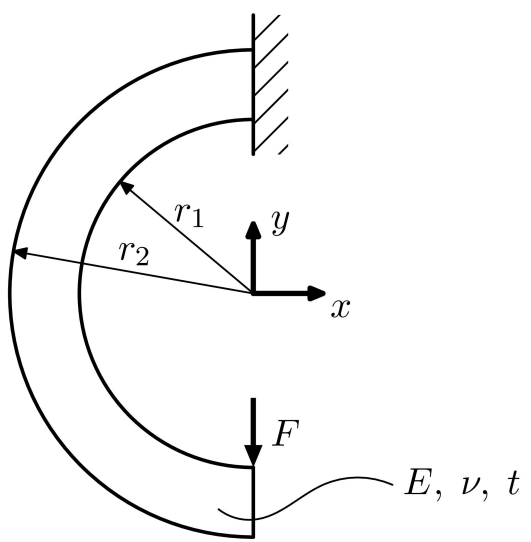

Figure 9. The C-Cantilever $\left(E=70 \cdot 10^{9} ; \nu=0.33 ; r_{1}=0,4 ; r_{2}=0,55 ; t=1\right.$; $F=1 \cdot 10^{7}$ ).

Next, a C-Cantilever illustrated in Figure 9 is considered. The problem is solved with a single distorted finite p-element. With the restriction that the discretization only involves one single finite element, the whole domain has to be mapped to the reference frame $[-1,1]^{2}$. The $\xi$-direction is assumed to be in axial direction of the beam, while the $\eta$-coordinate constitutes the thickness direction. In the following only an isoparametric approximation of the geometry is applied, exploiting the same ansatz-functions as for the displacement field approximation. Figure 10 shows the von-Mises stresses and the displacement fields of the GLL-solutions for three combinations of $p_{\xi}$ and $p_{\eta}$. It can be seen clearly, that a typical beam bending stress state arises in the center of the cantilever, involving pressure stresses
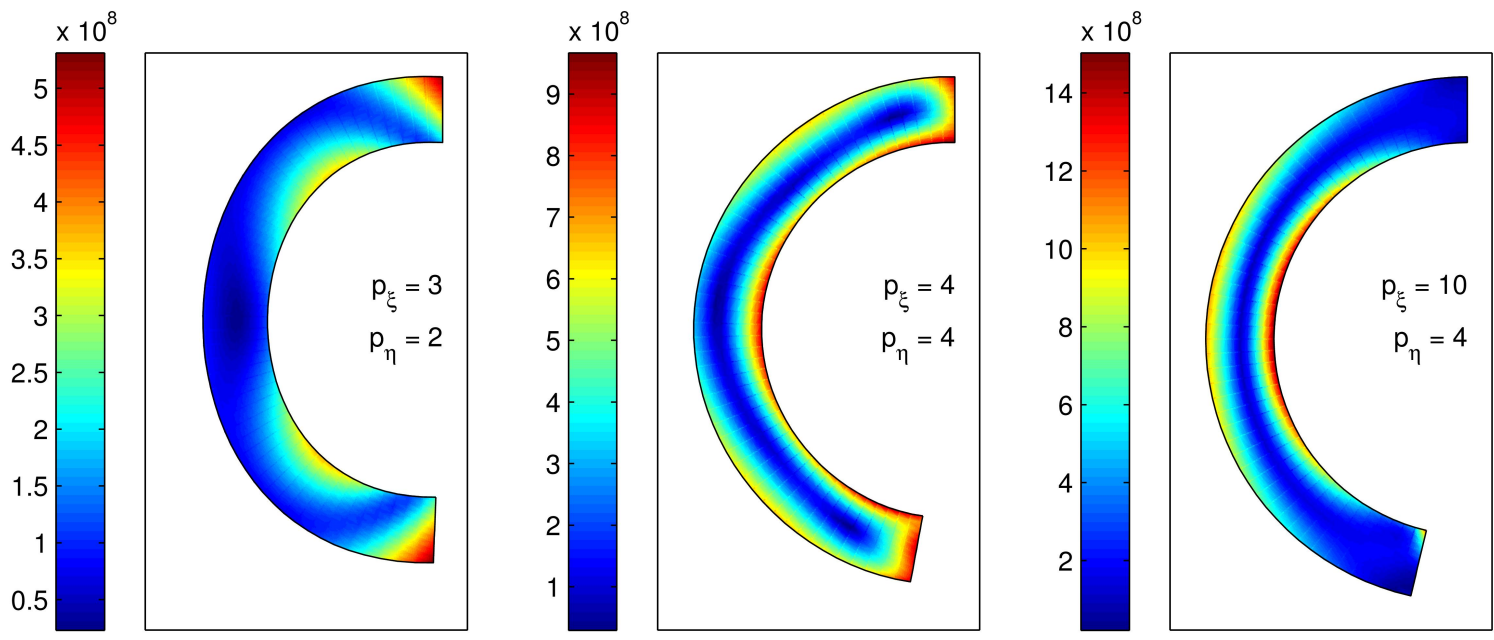

Figure 10. Von Mises stresses of the C-Cantilever for various degrees of shape functions regarding a GLL nodal distribution. 


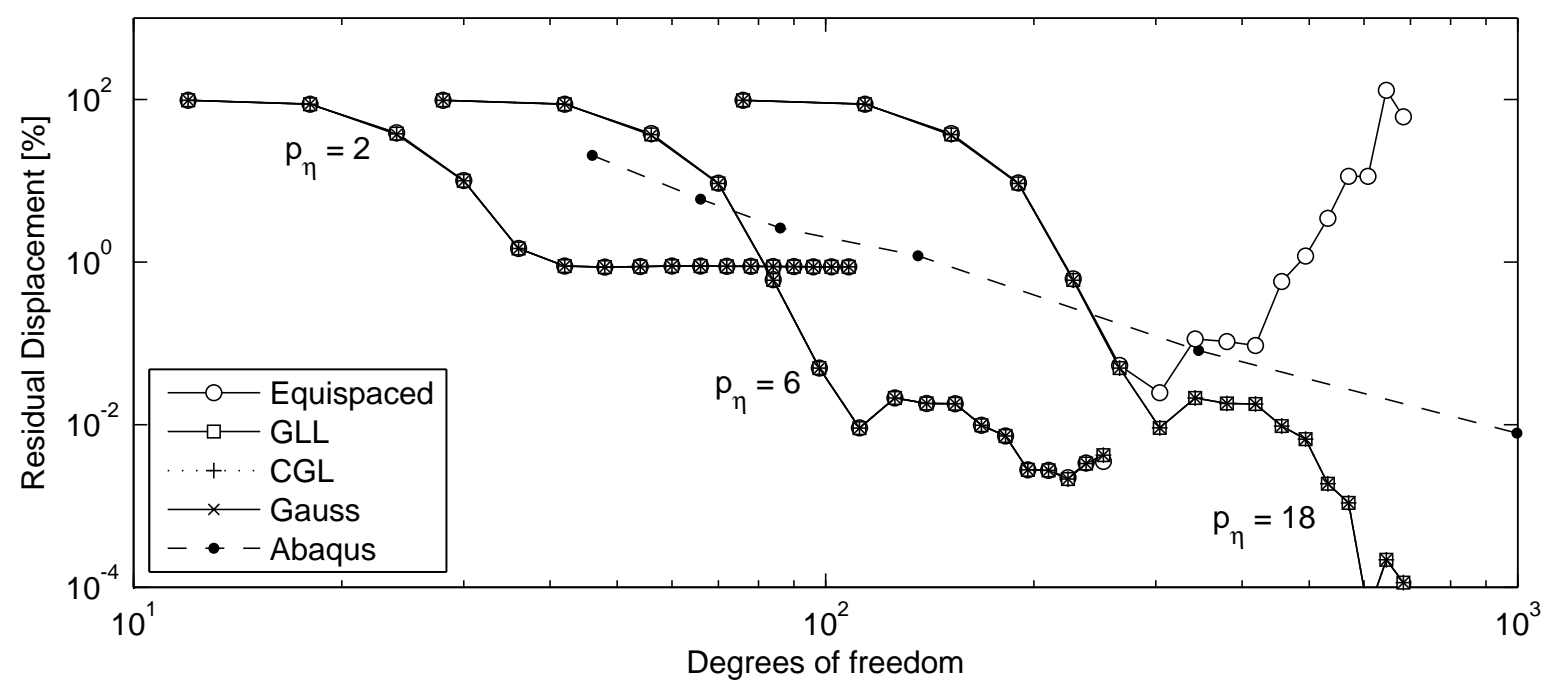

Figure 11. Residual $y$-displacement of the C-Cantilever's lower right corner at continuous raise of the polynomial degree $p_{\xi}=2,3, \ldots, 18$ and fixed $p_{\eta}$.

at the outer-surface and tensile stresses at the inner surface. The convergence plot in Figure 11 is based upon the displacement's $y$-component concerning the C-Cantilever's lower right corner. As in the former example, also here a residual value of

$$
\Delta u_{y}=\frac{\left\|u_{y}^{(p)}-u_{y}^{(h)}\right\|}{\left\|u_{y}^{(h)}\right\|}
$$

is computed by comparing it with a pseudo exact reference solution again calculated with Abaqus based on a very high mesh density ${ }^{4}$. In order to contrast the convergence behavior of the $p$-method, some Abaqus solutions based on standard square elements with quadratic shape functions and a standard $h$-refinement procedure with similar amounts of degrees of freedom have been added to this chart. Whereas these solutions converge algebraically, the p-element solutions exhibit a spectral convergence behavior. The latter is characterized by a monotonous decrease of the convergence curve's slope in a doubly logarithmic scaled diagram. Taking the graphs of the Lagrangian elements into account, it turns out, that beyond a certain point an increasing of $p_{\xi}$ does not improve the solution any more, which means that the curves end up on a plateau-like area. In the first case at $p_{\eta}=2$ this happens due to the fixed shape function degrees in thickness direction, which of course limits the solutions quality. The convergence disturbances of the other two graphs at $p_{\xi}>8$ can probably be attributed to the interference of an inexact approximation of the geometry as well as the minimization of the functional. But, like at the point loaded half-space with Dirichlet boundary conditions, also here the nodal distribution shows no effect on the convergence behavior. The solutions of the four analyzed nodal placements again almost coincide, except of the equispaced case, which starts to diverge at higher order polynomial degrees of the shape functions. This is again due to the rising condition number of the stiffness matrix in this case.

\footnotetext{
${ }^{4}$ This solution is based upon 14174 quadratic elements, resulting in a total of 86690 degrees of freedom.
} 


\subsection{Single distorted element: The mounted $L$}

The next static test is performed at a L-shaped component part being mounted at a ceiling as it is illustrated in Figure 12.

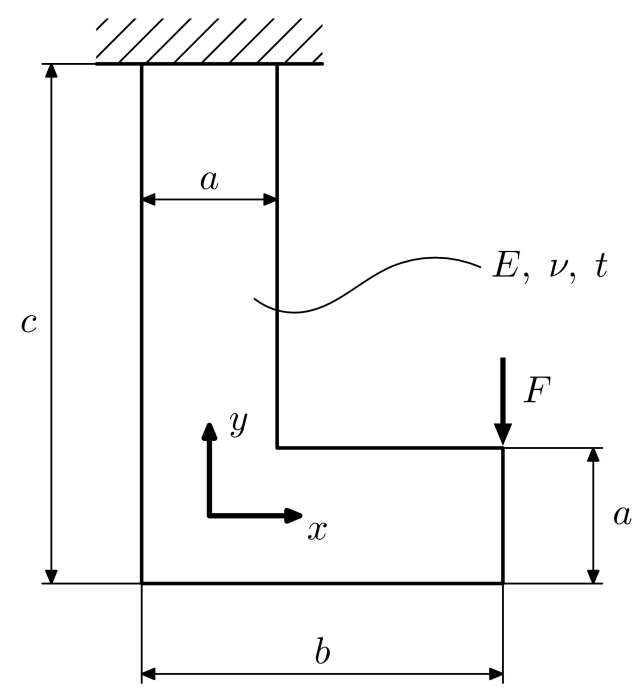

Figure 12. The mounted $\mathrm{L}\left(E=70 \cdot 10^{9} ; \nu=0.33 ; a=0.15 ; b=0.4 ; c=0.5\right.$, $\left.t=1 ; F=1 \cdot 10^{7}\right)$.

Even though it is not very recommendable to cover this domain by one single element, in the following exactly this is done in order to analyze the influence of the nodal distribution on an ill-posed geometrical approximation. Regarding the isoparametric mappings of the geometry it has to be distinguished between two nodal distributions, firstly, the one on the reference frame, and, secondly, the one on the physical component part as being displayed in Figure 13. While the former can generally be chosen from the set of the presented distributions in Section 3, the latter one has to be chosen in dependence of the given problem.

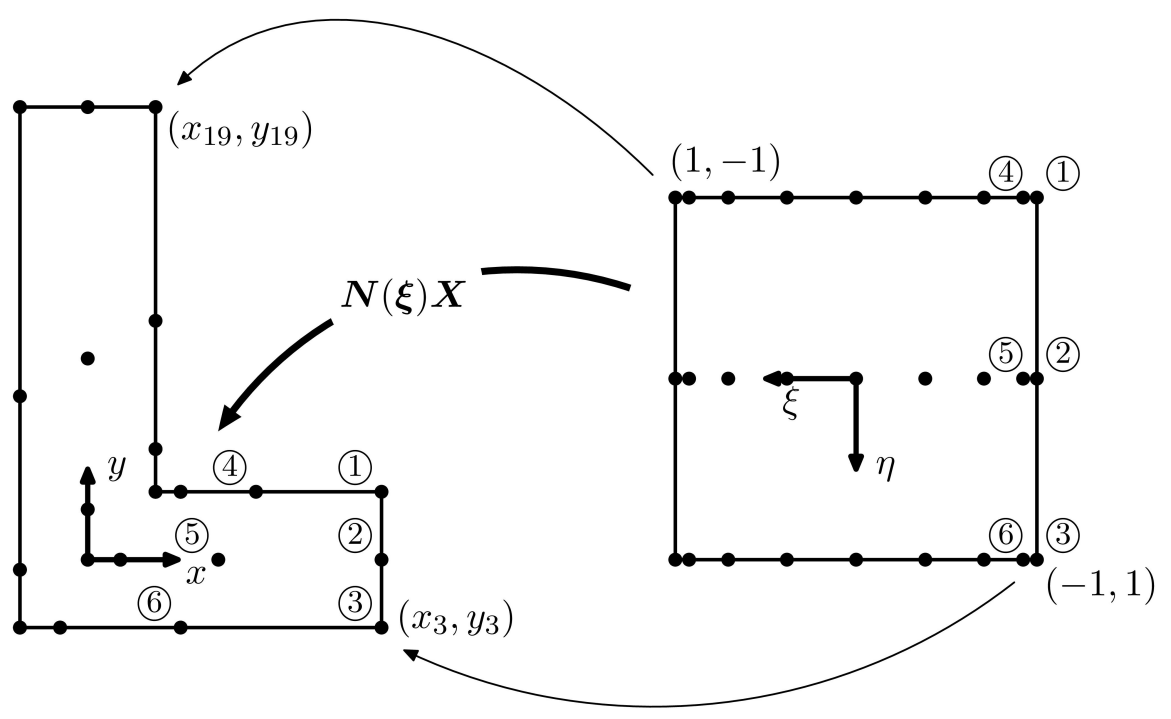

Figure 13 . The mounted L: Geometry mapping. 

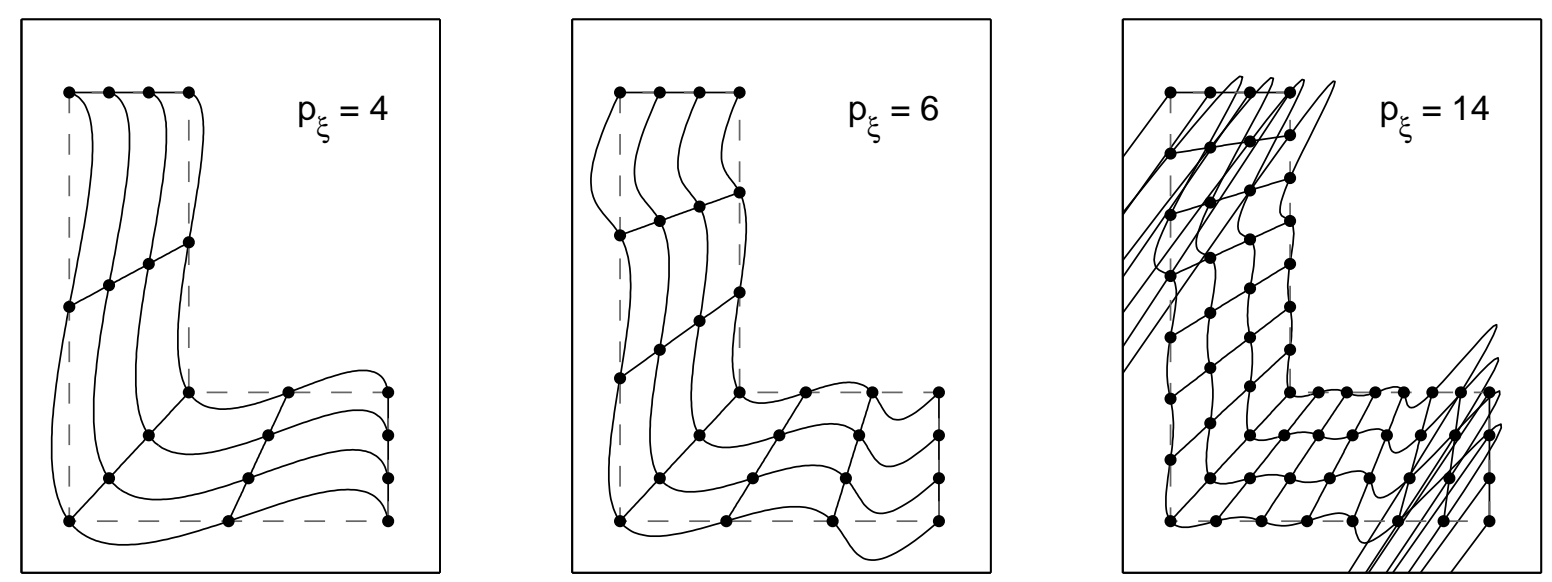

Figure 14. Geometry mapping of the mounted L with equally spaced nodes in both, the physical and the reference frame.
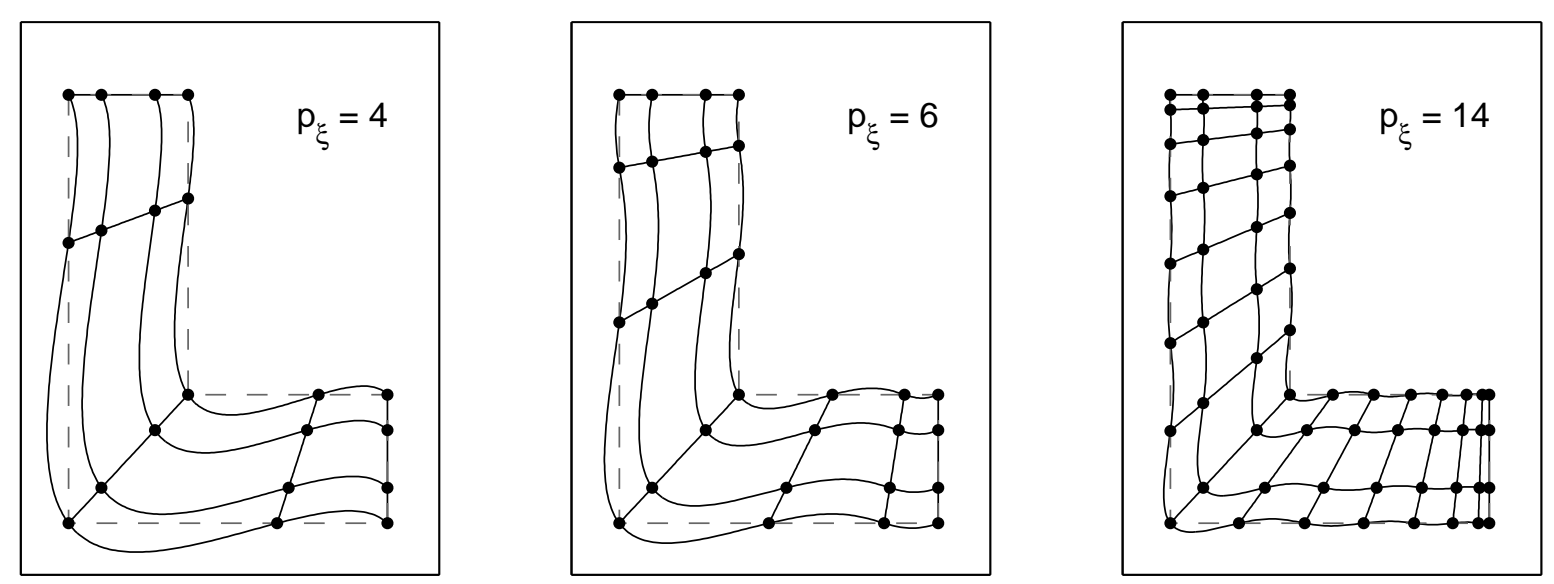

Figure 15. Geometry mapping of the mounted L with GLL-like distributed nodes in the physical frame, and GLL-distributed nodes in the reference frame.
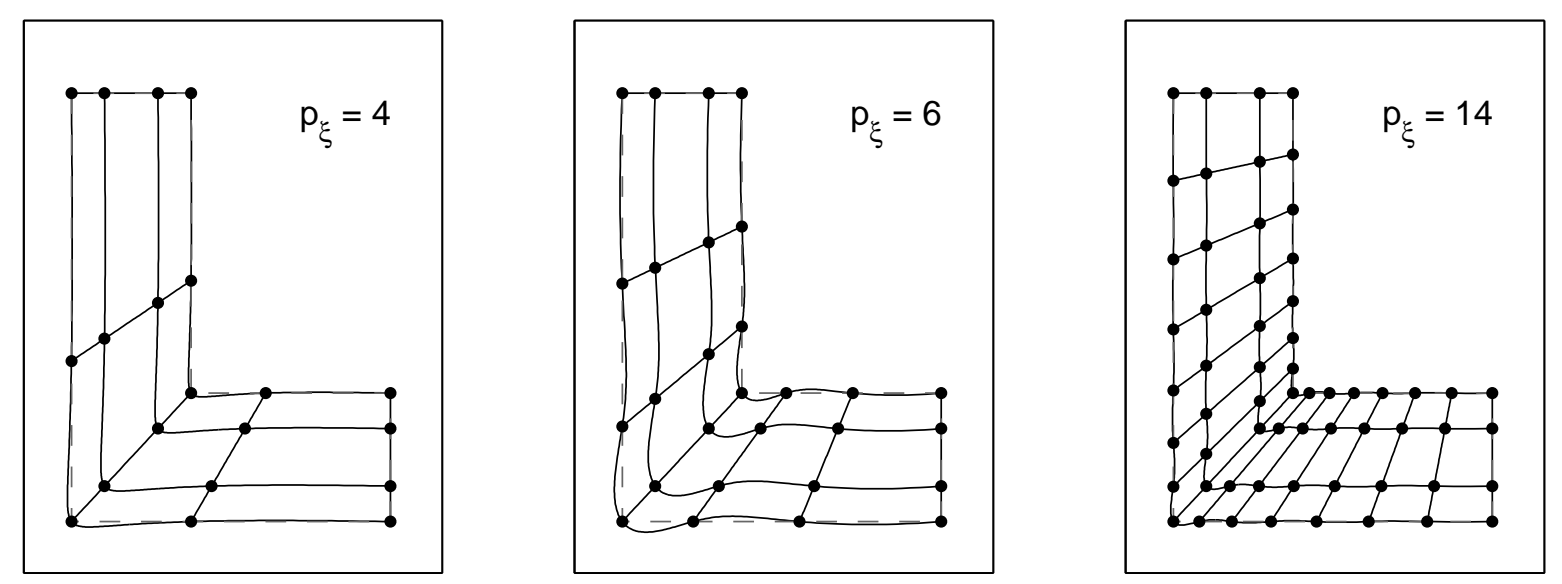

Figure 16. Geometry mapping of the mounted L with notch-centered grid-points in the physical frame and GLL-distributed nodes in the reference frame. 
In Figure 14-16 various options concerning these two nodal placements are illustrated utilizing the shape function degrees $p_{\xi}=4,6$ and 14, and $p_{\eta}=3$. Although in the prior example the shape of the C-Cantilever has been mapped well by utilization an equispaced nodal distribution in the reference frame, for the current example this turns out to be rather inapplicable, since for polynomial degrees $p_{\xi}>6$ strong Runge's oscillations occur. These oscillations are suppressed by switching to a GLL nodal distribution like in Figure 15. Even though in this case the geometrical mapping works at least, the L's notch is not very sharply mapped even at high polynomial degrees. Thus, in Figure 16 the reference nodes are maintained, while the physical nodes are centered to the corner. This option induces a very accurate description of the geometry, and, hence, is used for the subsequent analysis.
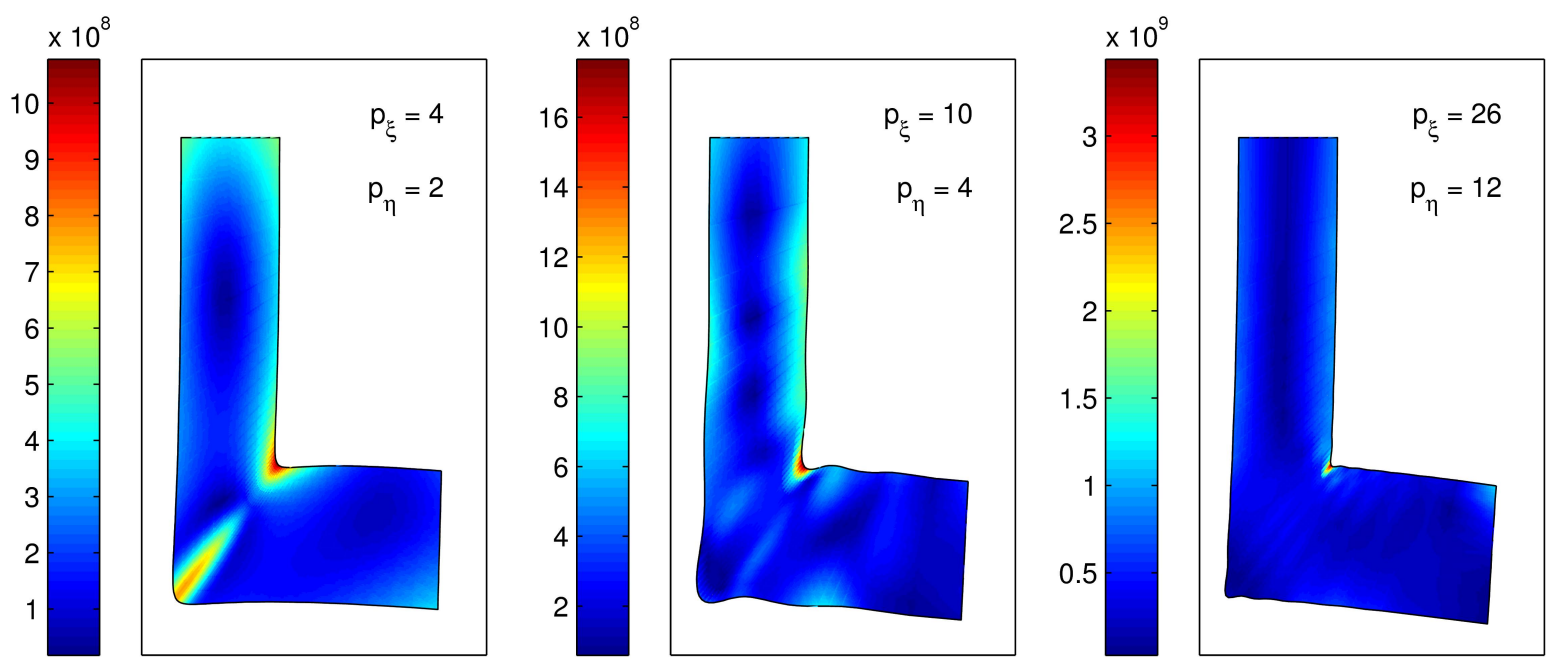

Figure 17. Von-Mises stresses of the mounted $\mathrm{L}$ for various shape function degrees.

In Figure 17 the resulting von-Mises stresses and displacements regarding a set of three combinations of $p_{\xi}$ and $p_{\eta}$ are plotted. Since the notch results in a singularity in this model, the stresses tend to infinity at this point as a p-refinement takes place. According to these plots, in Figure 18 the resulting $y$-displacements of the lower right corner are shown in dependence of the continuously increasing polynomial degrees $p_{\xi}$ and $p_{\eta}$. The ratio at which the two polynomial degrees are raising is $2: 1$, meaning that $p_{\xi}$ is chosen twice as big as $p_{\eta}$, while $p_{\eta}$ runs from 2 to 13 . This chart also contains an Abaqus solution ${ }^{5}$, which permits a rating of the solutions quality. It is evident, that only the GLL-grid with p-elements reaches the accuracy of the reference solution. Nevertheless, the CGL and the Gauss nodal distribution seem to converge also, but at a much slower rate. The equispaced solution is excluded from this diagram, since it diverges already at very low shape function orders, which is not a surprise due to the Runge's oscillations in the approximation of the geometry. In summary, it can be stated, that the nodal distribution used for the isoparametric mapping of a complicated geometry significantly impacts the solutions quality, which directly corresponds to the interpolation ability of the applied shape functions. 


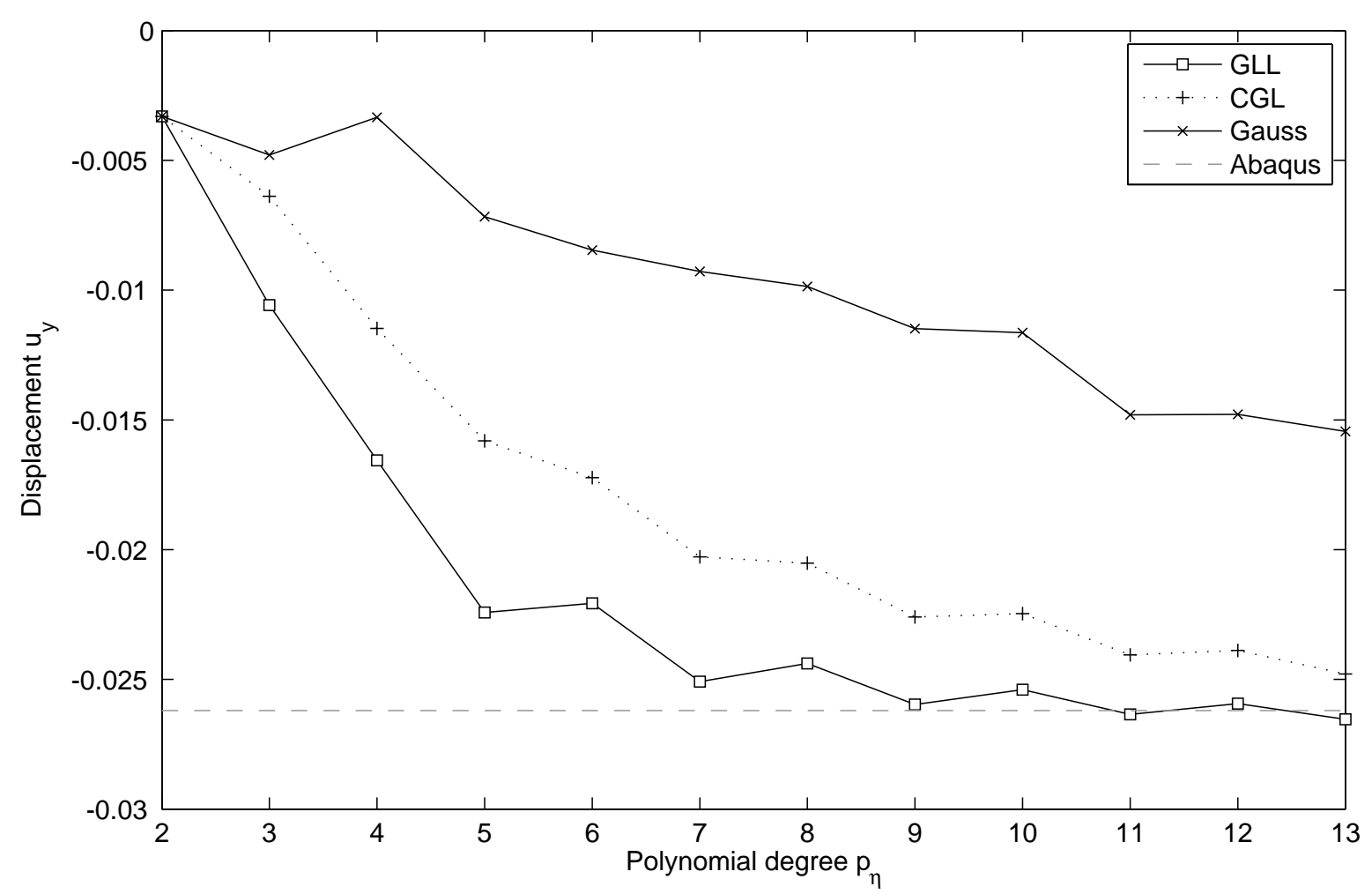

Figure 18. Convergence of the $y$-displacement of the lower right corner of the mounted L. The polynomial degrees are increased at a ratio of $2: 1$, meaning that $p_{\xi}=2 p_{\eta}$, with $p_{\eta}=2,3, \ldots, 13$.

\subsection{Single distorted element: The Flying S}

As a last example an unmounted S-shaped sheet is considered, as being illustrated in Figure 19. At first, the influence of the mass lumping in the GLL-case on the solution quality is investigated. At second, it is studied how the maximum stable step size of an explicit time integration scheme is altered by the nodal distribution at which the mass-lumping is performed. Like in all other test models, also here an isoparametric approximation of the geometry is applied, exploiting the same shape-functions as for the displacement field approximation. Therein the physical nodal distribution is adopted from the reference frame, as

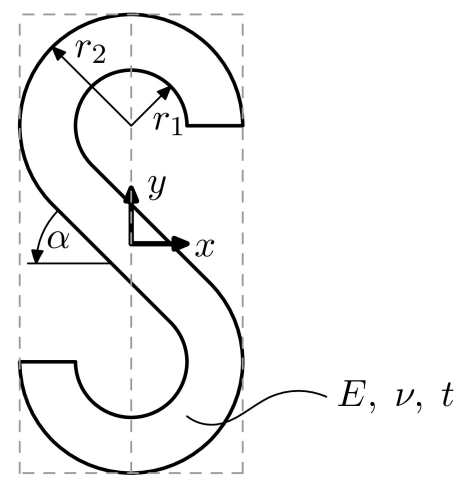

Figure 19. The flying $\mathrm{S}\left(E=70 \cdot 10^{9} ; \nu=0.33 ; \rho=2700 ; r_{1}=0.2 ; r_{2}=0.4 ; \alpha=45^{\circ}\right)$.

\footnotetext{
${ }^{5}$ This solution involves 7069 quadratic elements, which corresponds to a total of 21658 degrees of freedom.
} 

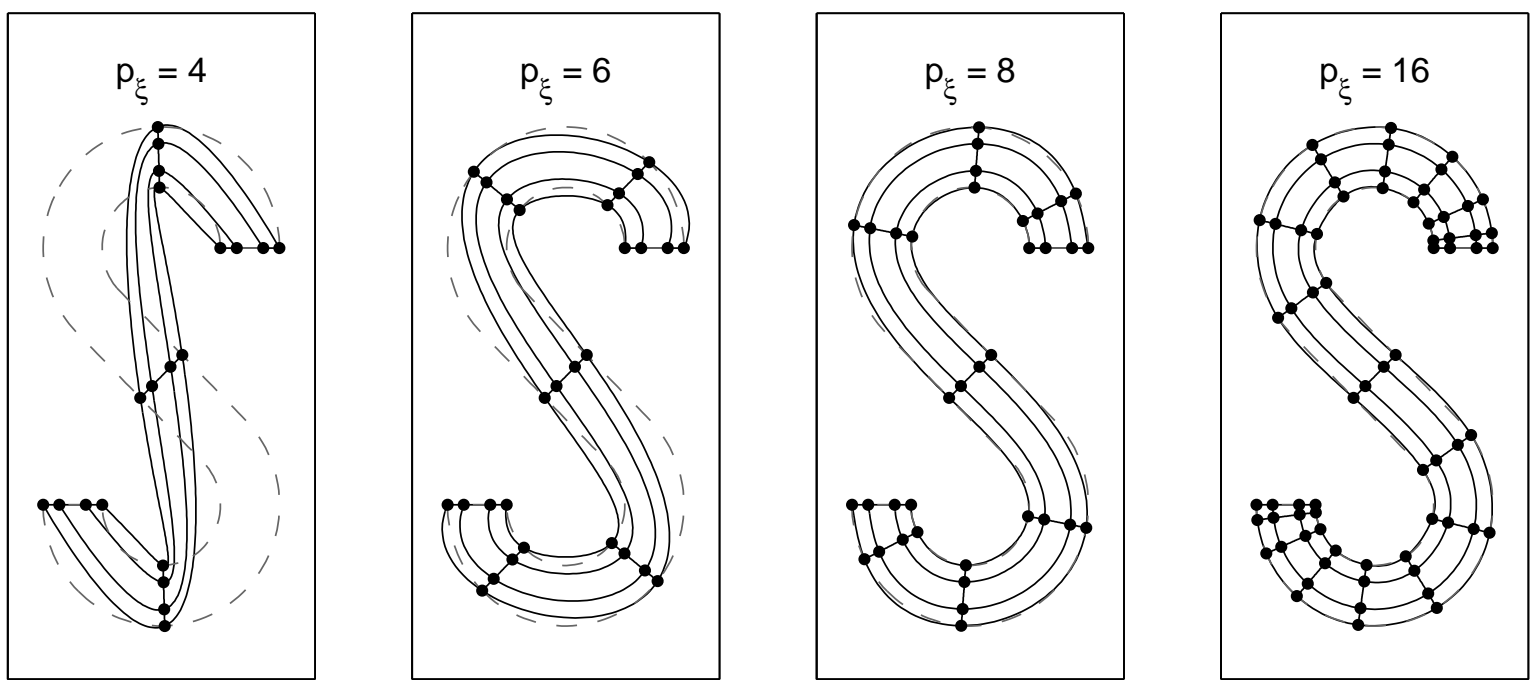

Figure 20. Geometry mapping of the flying S involving a GLL nodal distribution in the reference frame and a GLL-like nodal distribution in the physical frame. The polynomial degree $p_{\eta}$ is fixed to 3 .

it can be seen in Figure 20. According to the illustrated GLL nodal distribution the S-shape is mapped adequately well for polynomial degrees $p_{\xi} \geq 8$. In Figure 21 the first four eigenmodes and eigenfrequencies are displayed utilizing the GLL-element with fixed polynomial degrees $p_{\xi}=16$ and $p_{\eta}=8$, and a lumped mass matrix. Taking the first eigenfrequency $\omega_{1}$ into account involving a $p$-refinement based ansatz-function where the number of degrees of freedom along the $\mathrm{S}$ is twice as high as the degrees of freedom in thickness direction, the convergence behavior shown in Figure 22 is gained.
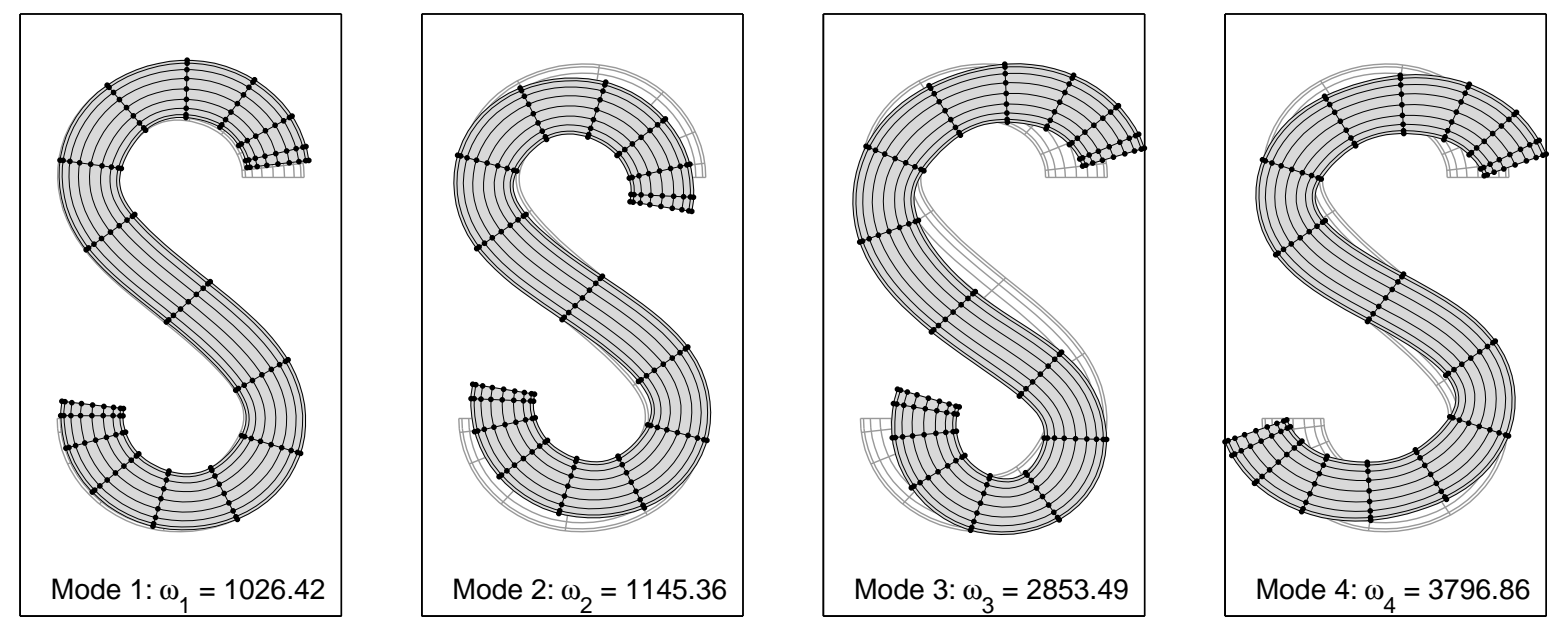

Figure 21. The first four eigenmodes and frequencies regarding one single GLL -element with completely integrated mass matrix at $p_{\xi}=16$ and $p_{\eta}=8$.

Evidently, even for this strongly distorted element the mass matrix lumping has hardly any impact on the calculated solutions. The computed eigenfrequencies of the lumped and the fully integrated mass matrices share the same magnitude of the errors, which is caused by the inexact description of the geometry. In conclusion, the mass matrix lumping of a distorted GLL-element does not necessarily worsen the solution conspicuously, and, hence, it is applicable under special care. 


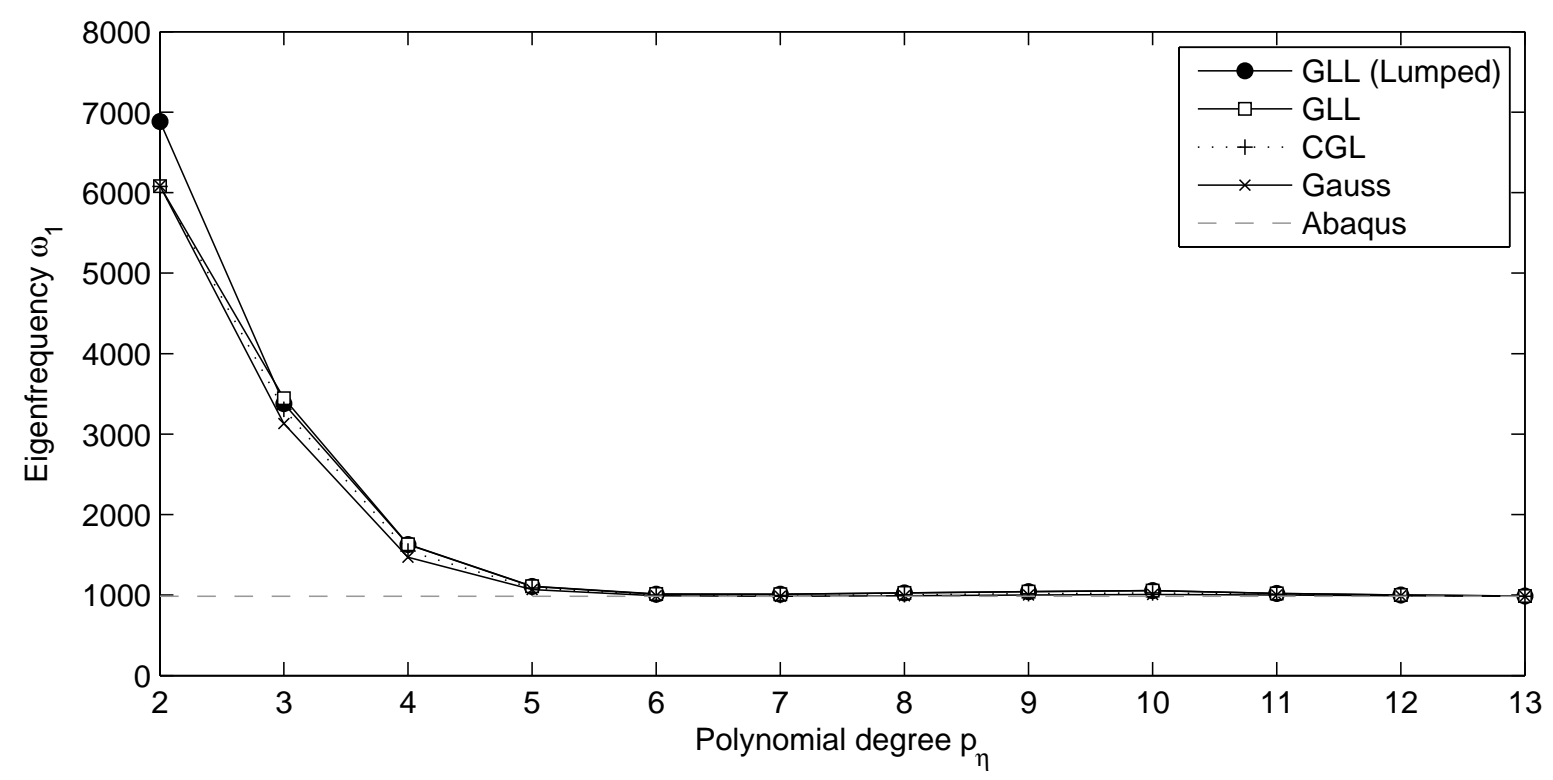

Figure 22. Convergence of the first eigenfrequency of the flying $\mathrm{S}$ at continuous $p$-refinement. The polynomial degrees are increased at a ratio $2: 1$, meaning $p_{\xi}=2 p_{\eta}$, while $p_{\eta}=2, \ldots, 13$.

Taking dynamic, transient problems into account the maximum stable time step-size is of crucial interest. Applying the central difference method the maximum eigenfrequency of the model influences the critical time step as [18]

$$
\Delta t \leq \Delta t_{c r i t}=\frac{2}{\sqrt{\lambda_{\max }\left(\boldsymbol{M}^{-1} \boldsymbol{K}\right)}} .
$$

According to the results plotted in Figure 23, the nodal distribution has hardly any influence

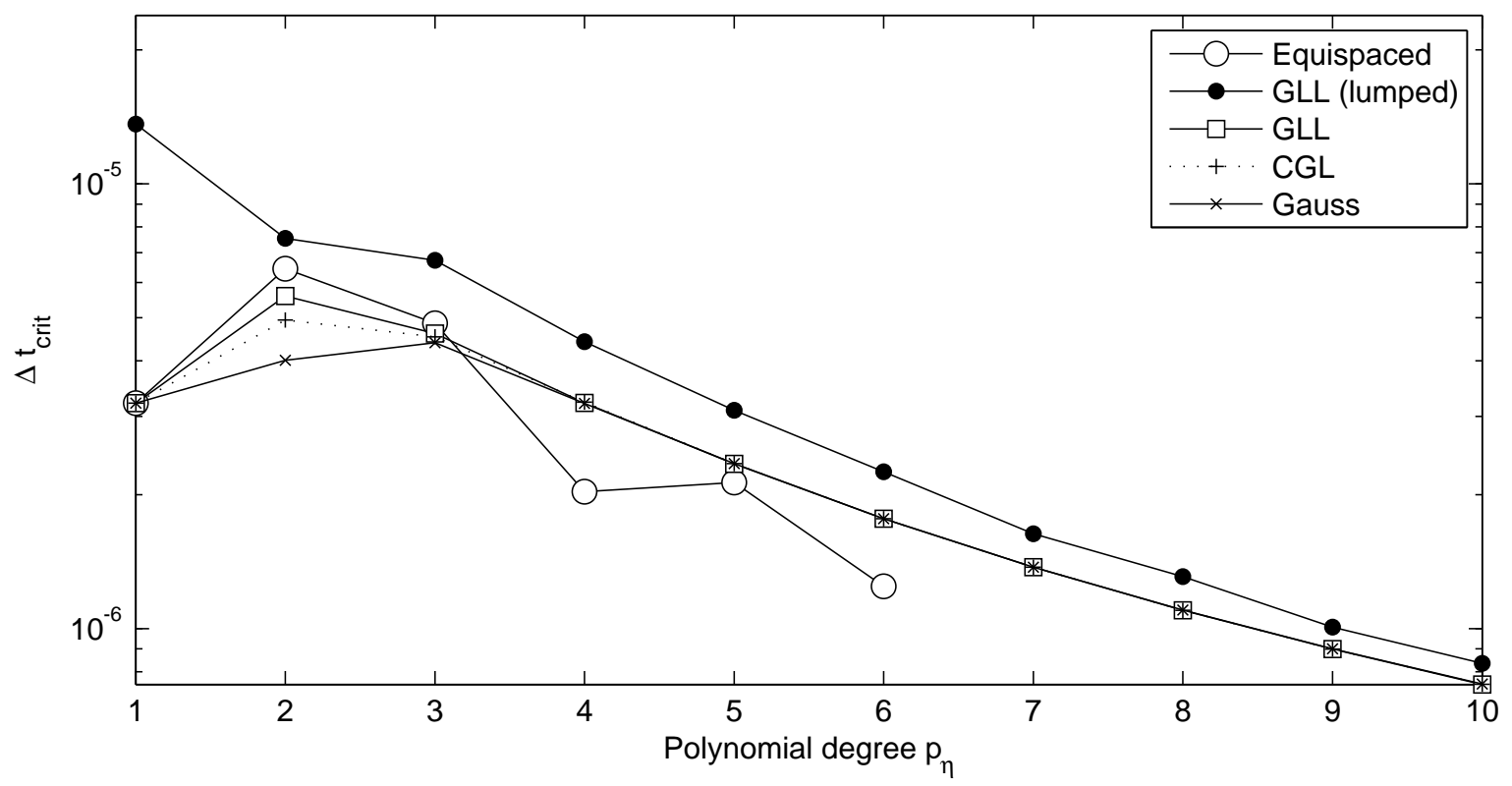

Figure 23. Maximum stable time step sizes of the flying $\mathrm{S}$ model at continuous $p$-refinement. The polynomial degrees are increased at a ratio $2: 1$, meaning $p_{\xi}=2 p_{\eta}$, while $p_{\eta}=2, \ldots, 10$. 
on the maximum stable time step-size neither. Mass matrix lumping in the GLL-case even increases this value. Hence, unlike at the h-method, where the highest nodal density of a model is a measure for the stability criterion, at the HOLP the higher nodal density at the boundary of elements does not cause lower stable time step sizes.

\section{CONCLUSIONS}

All performed tests have shown, that in general the nodal distribution does not affect the quality of the solution nor the convergence behavior of the applied algorithms directly. Significant differences are only observed for problems comprising an interpolation task, such as the geometrical descriptions or the treatment of the Dirichlet boundary conditions. Being strongly underlined at the example of the mounted L involving an ill-posed geometry approximation, the HOLP on an equispaced grid is fairly inapplicable due to the occurrence of strong Runge's oscillations. It is shown that in these cases the GLL-method converges fastest. The analysis of the condition number of the model with the point loaded half-space has revealed, that the mesh with EQ-grid elements results in very high condition values, which are much higher than the condition number from the three other investigated nodal placements. Consequently, the EQ-elements cannot be recommended to be applied in higher order finite element analysis. Although at such analysis the GLL-based finite element mesh is slightly better than the GP- and the CGL-based element meshes, the authors cannot recommend any of the three orthogonal polynomial based nodal placements as being best suited. The measured differences in most of the investigated tests cases are rather small. Only in cases where a diagonalized mass matrix is of advantage, we recommend the application of the GLL nodal placement, since to the best of the author's knowledge for the GP- or the CGL-based elements no such diagonalization technique is known.

\section{Acknowledgements}

The research is financially supported by the German Research Foundation (DFG) under grant GA480/13-2 and by the postgraduate program of the federal state of Saxony-Anhalt. This support is gratefully acknowledged.

\section{REFERENCES}

[1] Babuška I., Szabó B.A., Katz N. "The p-Version of the Finite Element Method". SIAM Journal on Numerical Analysis. Vol. 18, No. 3, 515-545, 1981.

[2] Babuška I., Suri M. "The p- and h-p Versions of the Finite Element Method, An Overview". Computer Methods in Applied Mechanics and Engineering. 80, 5-26, 1990.

[3] Szabó B.A., Babuška I. "Finite Element Analysis”. John Wiley and Sons. 1991.

[4] Solin P., Segeth K., Dolezel I. "Higher-Order Finite Element Methods". Chapman and Hall. 2004.

[5] Gopalakrishnan S., Chakraborty A., Mahapatra D.R. "Spectral Finite Element Method". Springer-Verlag. 2007.

[6] Boyd J.P. "Chebyshev and Fourier Spectral Methods”. Dover Publications. 2000. 
[7] Düster A. "High order finite elements for three-dimensional, thin-walled nonlinear continua". PhD-thesis at the Technical University of Munich. 2002.

[8] Duczek S. "Entwicklung eines finiten 3D-Schichtelementes nach der p-Methode für die Berechnung von Lamb-Wellen in Faserverbundstrukturen”. Diploma-thesis at the Ottovon-Guericke University of Magdeburg. 2010.

[9] Cottrell J.A., Reali A., Bazilevs Y., Hughes T.J.R. "Isogeometric analysis of structural vibrations". Computer Methods in Applied Mechanics and Engineering. Issues 41-43, 5257-5296, 2006.

[10] Willberg C., Gabbert U. "Development of a three dimensional piezoelectric isogeometric finite element for smart structure applications". Acta Mechanica, 2012, online available.

[11] Patera A.T. "A spectral element method for fluid dynamics: Laminar flow in a channel expansion". Journal of Computational Physics. 54, 468-488, 1984.

[12] Komatitsch D., Tromp J. "Spectral-element simulations of global seismic wave propagation - I. Validation". Geophysical Journal International. 149, 390-412, 2002.

[13] Schmicker D. "Development and testing of higher order finite elements based on Lagrange polynomials for the analysis of guided ultrasonic waves in thin-walled structures". Diploma-thesis at the Otto-von-Guericke University of Magdeburg, 2011.

[14] Zak A., Krawczuk M., Ostachowicz, W. "Propagation of in-plane waves in an isotropic panel with a crack". Finite Elements in Analysis and Design. 42, 929-941, 2011.

[15] Demaret P., Deville M.O. "Chebyshev Collocation Solutions of the Navier-Stokes Equations Using Multi-domain Decomposition and Finite Element Preconditioning”. Journal of Computational Physics. 95, 359-386, 1991.

[16] Zienkiewicz O.C., Taylor R.L. "The Finite Element Method: Volume 1, The Basis". Butterworth Heinemann. 2000.

[17] Pozrikidis C. "Introduction to Finite and Spectral Element Methods using MATLAB". Chapman and Hall. 2005.

[18] Bathe K.J. "Finite Element Procedures in Engineering Analysis". Springer-Verlag. 2002. 